\title{
Extraction of Affine Invariant Features Using Fractal
}

\author{
Jianwei Yang, ${ }^{1}$ Guosheng Cheng, ${ }^{1}$ and $\mathrm{Ming} \mathrm{Li}^{2}$ \\ ${ }^{1}$ School of Mathematics and Statistics, Nanjing University of Information Science and Technology, Nanjing 210044, China \\ ${ }^{2}$ School of Information Science and Technology, East China Normal University, No. 500, Dong-Chuan Road, Shanghai 200241, China
}

Correspondence should be addressed to Jianwei Yang; yjianw@hotmail.com

Received 19 March 2013; Accepted 29 April 2013

Academic Editor: Chen Wensheng

Copyright (c) 2013 Jianwei Yang et al. This is an open access article distributed under the Creative Commons Attribution License, which permits unrestricted use, distribution, and reproduction in any medium, provided the original work is properly cited.

\begin{abstract}
An approach based on fractal is presented for extracting affine invariant features. Central projection transformation is employed to reduce the dimensionality of the original input pattern, and general contour (GC) of the pattern is derived. Affine invariant features cannot be extracted from GC directly due to shearing. To address this problem, a group of curves (which are called shift curves) are constructed from the obtained GC. Fractal dimensions of these curves can readily be computed and constitute a new feature vector for the original pattern. The derived feature vector is used in question for pattern recognition. Several experiments have been conducted to evaluate the performance of the proposed method. Experimental results show that the proposed method can be used for object classification.
\end{abstract}

\section{Introduction}

The images of an object taken from different viewpoints often suffer from perspective distortions. For this reason, features extracted from the image of an object should be tolerant to an appropriate class of geometric transformation (such as translation, rotation, scaling, and shearing). A perspective transformation between two views can be approximated with an affine transformation if the object is planar and far away from the image plane [1]. Therefore, the extraction of affine invariant features plays a very important role in object recognition and has been found application in many fields such as shape recognition and retrieval $[2,3]$, watermarking [4], identification of aircrafts [5, 6], texture classification [7], image registration [8], and contour matching [9].

Many algorithms have been developed for affine invariant features extraction [10-12]. Based on whether the features are extracted from the contour only or from the whole shape region, the approaches can be classified into two main categories: region-based methods and contour-based methods. Contour-based methods provide better data reduction [13], but they are inapplicable to objects with several separable components. Region-based methods can achieve high accuracy but usually at the expense of high computational demands, for good overviews of the various techniques refer to [13-16]. Central projection transformation (CPT) [17] can be used to combine contour-based methods and region-based methods together. However, CPT cannot be used to extract affine invariant features directly. In this paper, we extract affine invariant features by integrating CPT and fractal.

The essential advantage of fractal technique descriptor is that it can greatly speed up computation [17]. Fractal, which is introduced by Mandelbrot [18], has been shown to be one of the most important scientific discoveries in the last century. It proposes a powerful tool for human being to explore the complexity. It can be used to model many classes of time-series data as well as images. The fractal dimension (FD) is an important characteristic of fractals; it contains information about their geometrical structure. Many applications of fractal concepts rely on the ability to estimate the FD of objects. In the area of pattern recognition and image processing, the FD has been used for image compression, texture segmentation, feature extraction [19, 20], and so forth. The utility of fractal to extract rotation invariant features has been invested in [17]. CPT is employed to reduce the dimensionality of the original pattern. A discrete wavelet transformation technique transforms the derived pattern into a set of subpatterns. Consequently, its FD is computed and has been used as the feature vectors. A satisfying classification rate has been achieved in the 


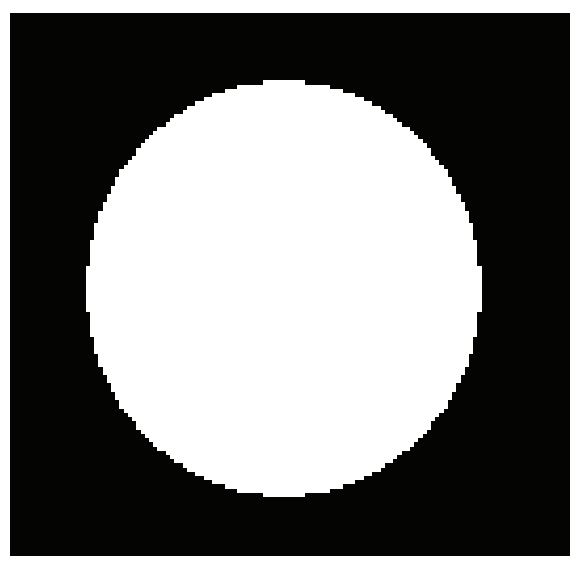

(a)

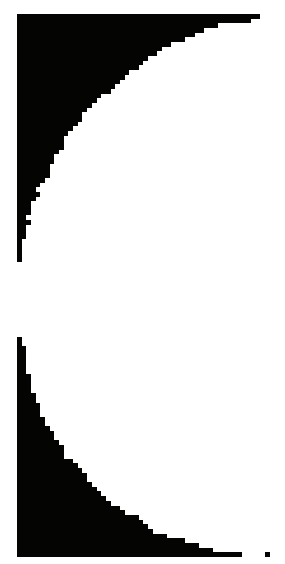

(b)

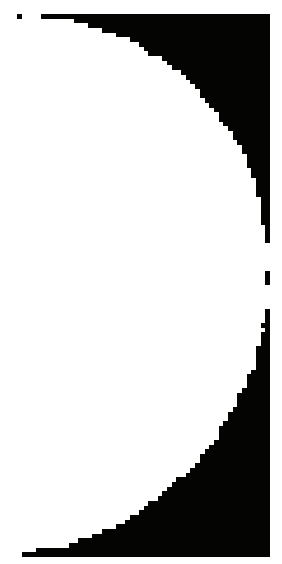

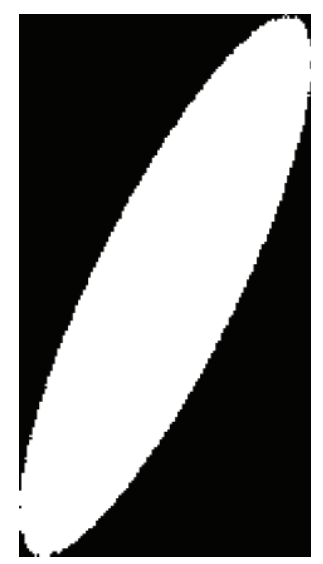

(c)

Figure 1: (a) An image of a circle. (b) A scaled version of circle in Figure 1(a). (c) An affine transformation version of circle in Figure 1(a).

recognition of rotated English letters, Chinese characters, and handwritten signatures. For more details, please refer to those papers.

However, the approach presented in [17] is hard to be used to extract invariant features for general affine transformation. A general affine transformation not only includes rotation, scaling, and translation but also includes shearing. That is to say, a circle may be transformed into an eclipse. Figure 1(a) is an image of a circle. Figure 1(b) is a scale and rotation version of circle in Figure 1(a). Figure 1(c) is an affine transformation version of circle in Figure 1(a). It can be calculated that FD of curve derived from the circle in Figure 1(a) by CPT is $f_{1}=1.4007$, FD of curve derived from the circle in Figure 1(b) by CPT is $f_{2}=1.4012$, while FD of curve derived from the eclipse in Figure $1(\mathrm{c})$ is $f_{3}=1.4244$. That is to say, FD can not be used to extract affine invariant features directly. To address this problem, a group of curves (which are called shift curves) are constructed from the closed curve derived by CPT in this paper. FDs of these curves can readily be computed and constitute a new feature vector for the original pattern. Several experiments have been conducted to evaluate the performance of the proposed method. Experimental results show that the constructed affine invariant feature vector can be used for object classification.

The rest of the paper is organized as follows. In Section 2, some basic concepts about CPT are introduced. The method for the extraction of affine invariant features is provided in Section 3. The performance of the proposed method is evaluated experimentally in Section 4. Finally, some conclusion remarks are provided in Section 5.

\section{CPT and Its Properties}

This section is devoted to providing some characteristics of CPT. In CPT, any object can be converted to a closed curve of the object by taking projection along lines from the centroid with different angles. Consequently, any object can be transformed into a single contour. In addition, the derived single contour also has affine property.
2.1. The CPT Method. Firstly, we translate the origin of the reference system to the centroid of the image. To perform CPT, the Cartesian coordinate system should be transformed to polar coordinate system. Hence, the shape $I(x, y)$ can be represented by a function $f$ of $r$ and $\theta$, namely, $I(x, y)=$ $f(r, \theta)$, where $r \in[0, \infty)$ and $\theta \in[0,2 \pi)$. After the transformation of the system, the CPT is performed by computing the following integral:

$$
g(\theta)=\int_{0}^{\infty} f(r, \theta) d r
$$

where $\theta \in[0,2 \pi)$.

Definition 1. For an angle $\theta \in \mathbb{R}, g(\theta)$ is given in (1) and $(\theta, g(\theta))$ denotes a point in the plane of $\mathbb{R}^{2}$. Letting $\theta$ go from 0 to $2 \pi$, then $\{(\theta, g(\theta)) \mid \theta \in[0,2 \pi)\}$ forms a closed curve. We call this closed curve the general contour (GC) of the object.

For an object $F$, we denote the GC extracted from it by $\mathrm{CPT}$ as $\partial_{F}$. The GC of an object has the following properties: single contour, affine invariant.

By (1), an angle $\theta \in[0,2 \pi)$ corresponds to a single value $g(\theta)$. Consequently, GC can be derived from any object by employing CPT. For instance, Figure 2(a) shows the image of Chinese character "Yang", which consists of several components. Figure 2(b) shows the GC of Figure 2(a). The object has been concentrated into an integral pattern, and a single contour has been extracted.

In real life, many objects consist of several separable components (such as Chinese character "Yang" in Figure 2(a)). Contour-based methods are unapplicable to these objects. By CPT, a single closed curve can be derived, and contour-based methods can be applied. Consequently, shape representation based on GC of the object may provide better data reduction than some region-based methods.

2.2. Affine Invariant of GC. An affine transformation $\mathbf{A}$ of coordinates $\mathbf{x} \in \mathbb{R}^{2}$ is defined as

$$
\mathbf{x}^{\prime}=\mathbf{A x}+\mathbf{b}
$$




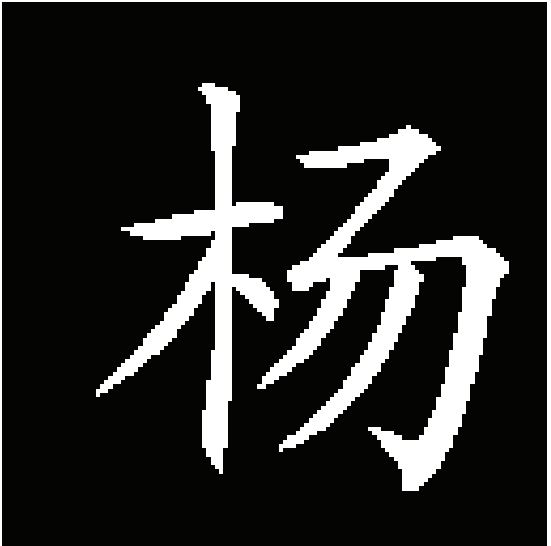

(a)

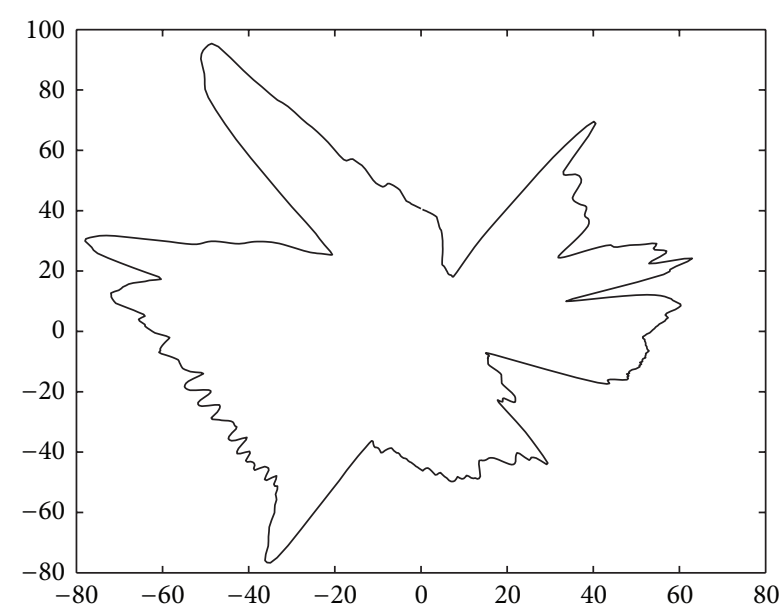

(b)

Figure 2: (a) The Chinese character "Hai". (b) GC derived from (a).

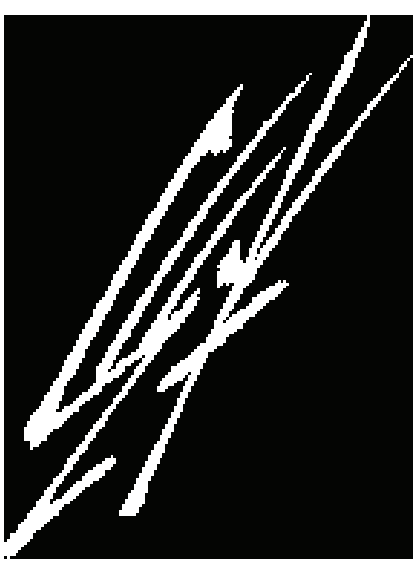

(a)

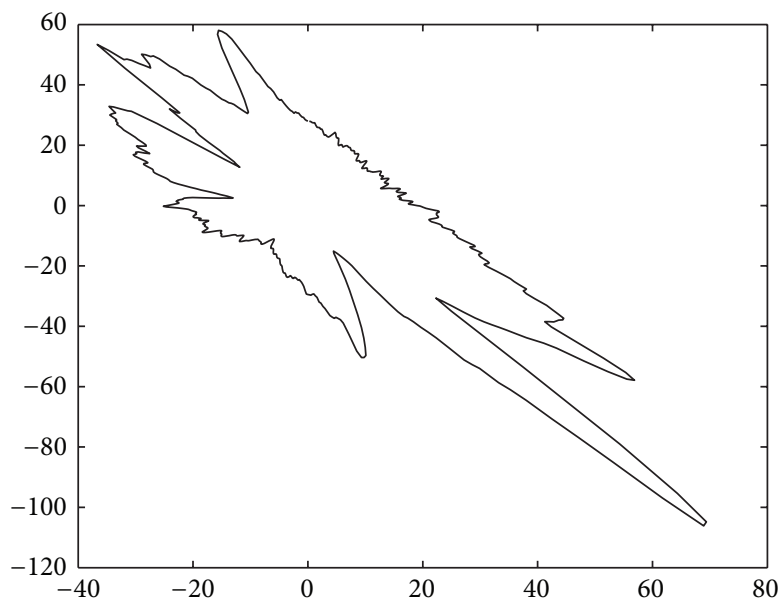

(b)

FIgURe 3: (a) An affine transformation version of Figure 2(a). (b) GC derived from (a).

where $\mathbf{b}=\left(\begin{array}{l}b_{1} \\ b_{2}\end{array}\right) \in \mathbb{R}^{2}$ and $\mathbf{A}=\left(\begin{array}{ll}a_{11} & a_{12} \\ a_{21} & a_{22}\end{array}\right)$ is a two-by-two nonsingular matrix with real entries.

Affine transformation maps parallel lines onto parallel lines and intersecting lines into intersecting lines. Based on this fact, it can be shown that the GC extracted from the affine-transformed object by CPT is also an affinetransformed version of GC extracted from the original object.

Consider two objects $F$ and $F^{\prime}$ are related by an affine transformation $\mathbf{A}$ :

$$
F^{\prime}=\left\{\mathbf{x}^{\prime} \mid \mathbf{x}^{\prime}=\mathbf{A} \mathbf{x}+\mathbf{b}, \mathbf{x} \in F\right\} .
$$

Then $\partial_{F}$ and $\partial_{F^{\prime}}$, GCs of $F$ and $F^{\prime}$, are related by the same affine-transformation $\mathbf{A}$ :

$$
\partial_{F}^{\prime}=\left\{\mathbf{x}^{\prime} \mid \mathbf{x}^{\prime}=\mathbf{A x}+\mathbf{b}, \mathbf{x} \in \partial_{F}\right\} .
$$

For example, Figure 3(a) shows an affine transformed version of Figures 2(a) and 3(b) shows the GC derived from Figure 3(a). Observing the two GCs in Figures 2(b) and $3(\mathrm{~b})$, we can see that CPT not only represents the distribution information of the object but also preserves the affine transformation signature.

Therefore, to see whether an object $\Theta$ is the affine transform version of $F$, we just need to check if $\partial \Theta$, the GC of $\Theta$, is the same affine-transformed version of $\partial_{F}$. We extract affine invariant features using fractal from GC of the object.

\section{Extraction of Affine Invariant Features Using Fractal}

By CPT, a closed curve can be derived from any object. In order to extract affine invariant features from the derived GC, the GC should firstly be parameterized. Thereafter, shift curves are constructed from the parameterized GC. Consequently, divider dimensions of these curves are computed to form feature vectors. 


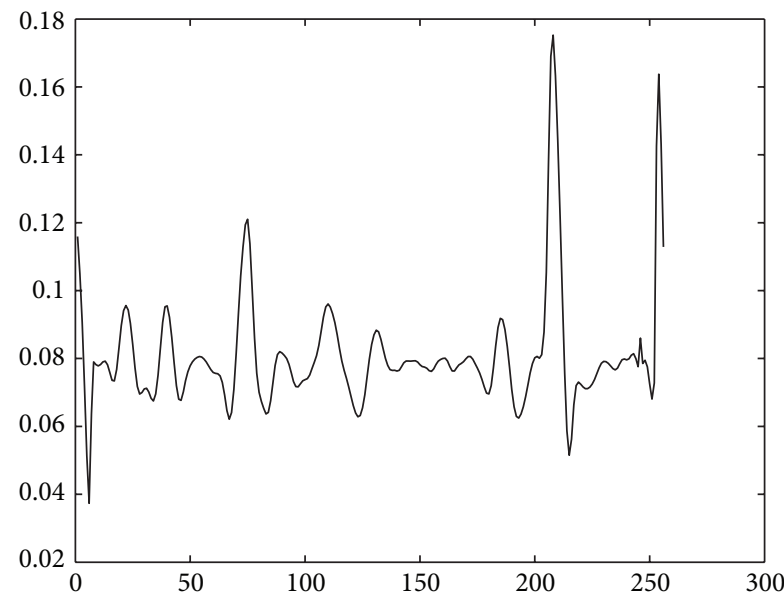

(a)

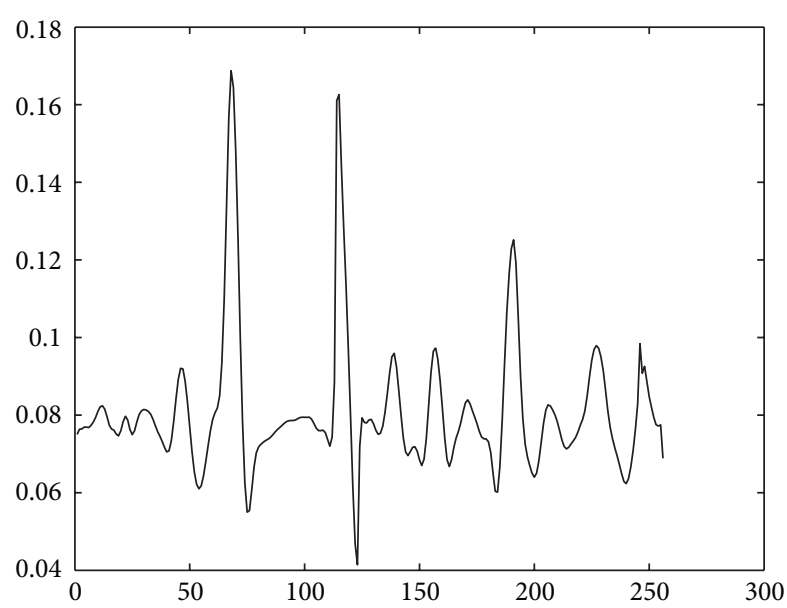

(b)

FIgURE 4: (a) 10-shift curve of the Chinese character given in Figure 2(a). (b) 10-shift curve of the Chinese character given in Figure 3(a).

3.1. Affine Invariant Parameterization. GC should be parameterized to establish one-to-one relation between points on GC of the original object and those on GC of its affine transformed version.

There are two parameters which are linear under an affine transformation: the affine arc length [21], and the enclosed area [22]. These two parameters can be made completely invariant by simply normalizing them with respect to either the total affine arc length or the enclosed area of the contour. In the discrete case, the derivatives can be calculated using finite difference equations. The curve normalization approach used in this paper is the same as the method given in [23]. In the experiments of this paper, GC is normalized and resampled such that $N=256$.

Suppose that GC of the object has been normalized and resampled. Furthermore, we suppose that the starting point on GC of the original object and that on GC of the affinetransformed version of the original object are identical. Then, a parametric point $\mathbf{x}(\sigma)=[x(\sigma), y(\sigma)]^{T}$ on GC of the original object and a parametric point $\widetilde{\mathbf{x}}(\widetilde{\sigma})=[\widetilde{x}(\sigma), \widetilde{y}(\sigma)]^{T}$ on GC of its affine transformed version satisfy the following equation:

$$
\begin{aligned}
& \tilde{x}(\widetilde{\sigma})=a_{11} x(\sigma)+a_{12} y(\sigma)+b_{1} \\
& \tilde{y}(\widetilde{\sigma})=a_{21} x(\sigma)+a_{22} y(\sigma)+b_{2} .
\end{aligned}
$$

3.2. Shift Curves. In this part, we will derive invariant features from the normalized GC. Let $[x(\sigma), y(\sigma)]$ and $[\tilde{x}(\widetilde{\sigma})$, $\tilde{y}(\widetilde{\sigma})]$ be the parametric equations of two GCs derived from objects that differ only by an affine transformation. For simplicity, in this subsection, we assume that the starting points on both GCs are identical. After normalizing and resampling, there is a one-to-one relation between $\sigma$ and $\widetilde{\sigma}$. We use the object centroid as the origin, then translation factor $\mathbf{b}$ is elimated. Equation (2) can be written in matrix form as $\widetilde{\mathbf{x}}(\widetilde{\sigma})=\mathbf{A x}(\sigma)$.
Letting $\gamma$ be an arbitrary positive constant, then $[x(\sigma+$ $\gamma), y(\sigma+\gamma)]$ is a shift version of $[x(\sigma), y(\sigma)]$. We denote $M_{0}$ as the zero moment of the object:

$$
M_{0}=\iint I(x, y) d x d y .
$$

We define the following function:

$$
C_{\gamma}(\sigma)=\frac{1}{M_{0}}|x(\sigma) y(\sigma+\gamma)-y(\sigma) x(\sigma+\gamma)| .
$$

We call $C_{\gamma}(\sigma)$ as $\gamma$ shift curve of the object. Figure 4(a) shows a 10-shift curve of the Chinese character given in Figure 2(a). As a result of normalizing and resampling, $[x(\sigma), y(\sigma)]$, $[\tilde{x}(\widetilde{\sigma}), \tilde{y}(\widetilde{\sigma})]$ and $[x(\sigma+\gamma), y(\sigma+\gamma)],[\tilde{x}(\widetilde{\sigma}+\gamma), \tilde{y}(\widetilde{\sigma}+\gamma)]$ satisfy the following equation:

$$
\begin{gathered}
\tilde{x}(\widetilde{\sigma})=a_{11} x(\sigma)+a_{12} y(\sigma), \\
\tilde{y}(\widetilde{\sigma})=a_{21} x(\sigma)+a_{22} y(\sigma), \\
\tilde{x}(\widetilde{\sigma}+\gamma)=a_{11} x(\sigma+\gamma)+a_{12} y(\sigma+\gamma), \\
\tilde{y}(\widetilde{\sigma}+\gamma)=a_{21} x(\sigma+\gamma)+a_{22} y(\sigma+\gamma) .
\end{gathered}
$$

It follows that

$$
\begin{aligned}
\widetilde{C}_{\gamma}(\widetilde{\sigma}) & =\frac{|\tilde{x}(\widetilde{\sigma}) \tilde{y}(\widetilde{\sigma}+\gamma)-\tilde{y}(\tilde{\sigma}) \tilde{x}(\widetilde{\sigma}+\gamma)|}{\widetilde{M}_{0}} \\
& =\frac{\operatorname{det}(\mathbf{A})|x(\sigma) y(\sigma+\gamma)-y(\sigma) x(\sigma+\gamma)|}{\operatorname{det}(\mathbf{A}) M_{0}} \\
& =C_{\gamma}(\sigma) .
\end{aligned}
$$

In other words, $C_{\gamma}$ given in (7) is affine invariant.

Note that, after affine transformation, the starting point of GC is different. Figure 4(b) shows the 10-shift curve of the affine transformed version Chinese character given in Figure 3(a). We observe that the shift curve of an affine transformation version of the object (see Figure $4(\mathrm{~b})$ ) is a translated version of the shift curve of the original object (see Figure 4(a)). 
3.3. Computing Divider Dimension of Shift Curves. The FD is a useful method to quantify the complexity of feature details present in an image. In this subsection, we shall discuss the problem of computing the divider dimension of shift curves and, thereafter, use the computed divider dimension to construct a feature vector for the original two-dimensional pattern in question for pattern recognition.

Fractals are mathematical sets with a high degree of geometrical complexity, which can model many classes of time series data as well as images. The FD is an important characteristic of the fractals because it contains information about its geometric structures. When employing fractal analysis researchers typically estimate the dimension from an image. Of the wide variety of FDs in use, the definition of Hausdorff is the oldest and probably the most important. Hausdorff dimension has the advantage of being defined for any set and is mathematically convenient, as it is based on measures, which are relatively easy to manipulate. A major disadvantage is that in many cases it is hard to calculate or estimate by computation methods.

In general, the dimension of a set can be found by the equation

$$
D=\frac{\log (N)}{\log (1 / r)},
$$

where $D$ is the dimension and $N$ is the number of parts comprising the set, each scaled down by a ratio $r$ from the whole [18].

In what follows, we use the notion of divider dimension of a nonself-intersecting curve (see $[24,25]$ etc.). Suppose that $C$ is a nonself-intersecting curve and $\delta>0$. Let $M_{\delta}(C)$ be the maximum number of ordered sequence of points $x_{0}, x_{1}, \ldots, x_{M}$ on curve $C$, such that $\left|x_{k}-x_{k-1}\right|=\delta$ for $k=1,2, \ldots, M$. The divider dimension $\operatorname{dim}_{D} C$ of curve $C$ is defined as follows:

$$
\operatorname{dim}_{D} C=\lim _{\delta \rightarrow 0} \frac{\log M_{\delta}(C)}{-\log \delta},
$$

where $\left|x_{k}-x_{k-1}\right|$ represents the magnitude of the difference between two vectors $x_{k}$ and $x_{k-1}$.

It should also be mentioned that $x_{M}$ is not necessarily the end point of curve $C, x_{T}$, but $\left|x_{T}-x_{M}\right|<\delta$. Furthermore, $\left(M_{\delta}(C)-1\right) \delta$ may be viewed as the length of curve $C$ as measured using a pair of dividers that are set $\delta$ distance apart.

Since the divider dimension of nonself-intersecting curves is asymptotic values, we derive their approximations based on the following expression in our experiments:

$$
\frac{\log M_{\delta}(C)}{-\log \delta}
$$

where $\delta$ is set small enough.

The divider dimension of shift curve in Figure 4(a) is 1.6168 and that of shift curve in Figure 4(b) is 1.6195. In the experiments of this paper, divider dimensions of shift curves $\gamma=1,2, \ldots, 256$ are computed. The feature vector is constituted

$$
V_{\text {fea }}=\left(\mathrm{FD}_{1}, \mathrm{FD}_{2}, \ldots, \mathrm{FD}_{255}\right)^{T}
$$

\section{Experiment}

In this section, we evaluate the discriminate ability of the proposed method. In the first experiment, we examine the proposed method by using some airplane images. Object contours can be derived from these images. In the second experiment, we evaluate the discriminate ability of the proposed method by using some Chinese characters. These characters have several separable components, and contours are not available for these objects.

In the following experiments, the classification accuracy is defined as

$$
\eta=\frac{\delta}{\lambda} \times 100 \%
$$

where $\delta$ denotes the number of correctly classified images and $\lambda$ denotes the total number of images applied in the test. Affine transformations are generated by the following matrix [5]:

$$
A=k\left(\begin{array}{cc}
\cos \theta & -\sin \theta \\
\sin \theta & \cos \theta
\end{array}\right)\left(\begin{array}{ll}
a & b \\
0 & \frac{1}{a}
\end{array}\right),
$$

where $k, \theta$ denote the scaling and rotation transformation, respectively, and $a, b$ denote the skewing transformation. To each object, the affine transformations are generated by setting the parameters in (15) as follows: $k \in\{0.8,1.2\}, \theta \in\left\{30^{\circ}, 90^{\circ}, 150^{\circ}, 210^{\circ}, 270^{\circ}, 330^{\circ}\right\}, b \in$ $\{-3 / 2,-1,-1 / 2,0,1 / 2,1,3 / 2\}$, and $a \in\{1,2\}$. Therefore, each image is transformed 168 times.

4.1. Binary Image Classification. In these experiments, we examine the discrimination power of the proposed method using 40 Chinese characters shown in Figure 5. These Chinese characters are with regular script font, and the images have size $128 \times 128$ in the experiments. We observe that some characters in this database have the same structures, but the number of strokes or the shape of specific stokes may be a little different. Some characters consist of several separable components. As aforementioned, each character image is transformed 140 times. That is to say, the test is repeated 5600 times. Experiments on these Chinese characters in Figure 5 and their affine transformations show that $98.14 \%$ accurate classification can be achieved by the proposed method.

The images are sometimes noised for reasons in many real-life recognition situations. The robustness of the proposed method is tested using binary image in this part. We add salt and pepper noise to the transformed binary images. We compare the proposed method with two regionbased methods, namely, the AMIs and MSA. The comparative methods are described in [26, 27], and these methods are implemented as discussed in those articles. 3 AMIs and 29 MSA invariants are selected for recognition. The nearest neighbor classifier is applied for AMIs and MSA methods. We firstly add the salt and pepper noise with intensities varying from 0.005 to 0.03 to the transformed images.

Table 1 shows the classification accuracies of all methods in the corresponding noise degree. We can observe that 

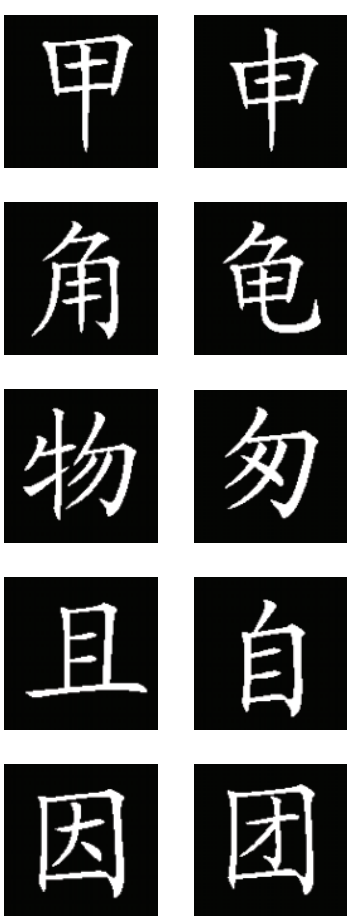
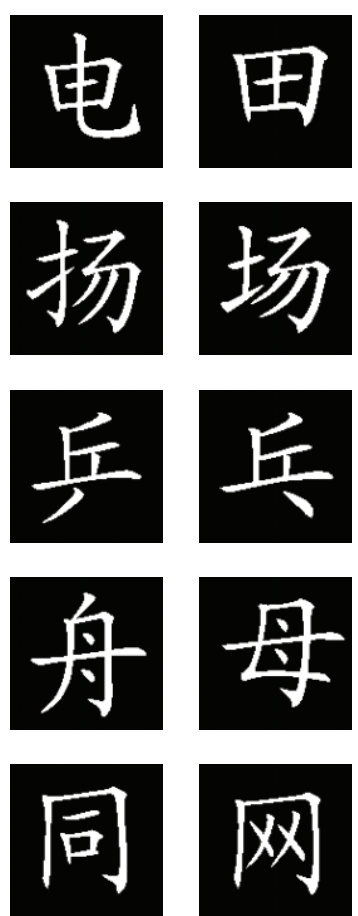
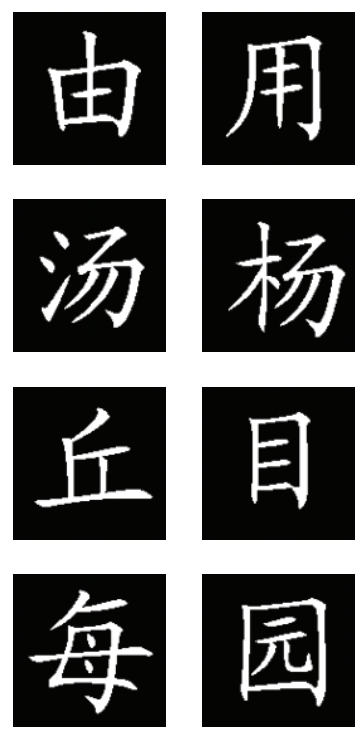
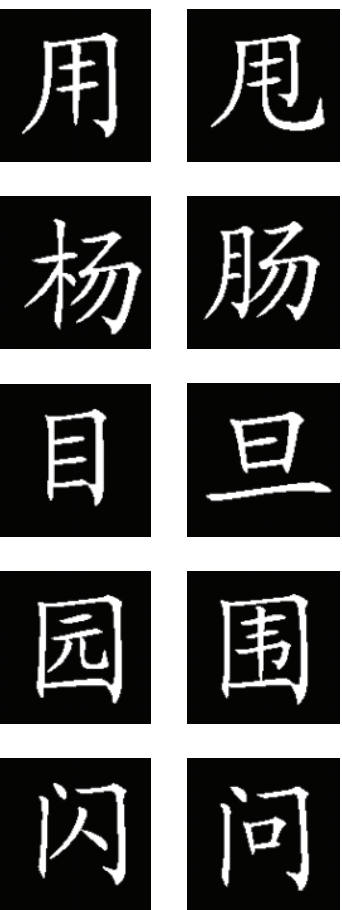
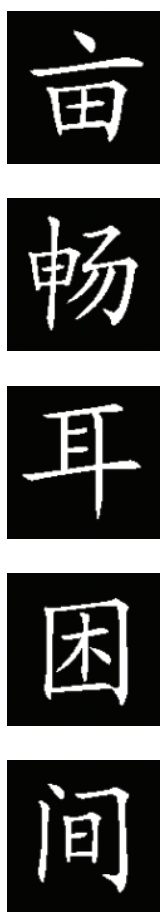

FIGURE 5: Test characters used in the second experiments.

TABLE 1: Classification accuracies of AMIs, MSA, and the proposed method for Chinese characters in case of affine transformation and different intensities of salt and pepper noise.

\begin{tabular}{lccccccc}
\hline Noise degree & 0 & 0.005 & 0.010 & 0.015 & 0.020 & 0.025 & 0.030 \\
\hline AMIs & $91.70 \%$ & $45.50 \%$ & $34.82 \%$ & $28.54 \%$ & $23.82 \%$ & $19.89 \%$ & $17.77 \%$ \\
MSA & $94.48 \%$ & $90.63 \%$ & $73.57 \%$ & $53.80 \%$ & $38.16 \%$ & $26.86 \%$ & $19.88 \%$ \\
Our method & $98.14 \%$ & $96.21 \%$ & $93.48 \%$ & $89.46 \%$ & $86.25 \%$ & $82.07 \%$ & $77.59 \%$ \\
\hline
\end{tabular}

the classification accuracy of AMIs decreases rapidly from noise-free condition to small noise degree. The classification accuracy decreases from $91.70 \%$ to less than $50 \%$ when the noise intensity is 0.010 . MSA performs much better than AMIs, but the results are not satisfying. To large noise degrees, the proposed method keeps high accuracies all the time.

4.2. Gray Image Classification. In this part, the well-known Columbia Coil-20 database [28], which contains 20 different objects shown in Figure 6, is applied in this experiment. To each object, the affine transformations are generated by setting the parameters in (15) as aforementioned. Therefore, each image is transformed 140 times. That is to say, the test is repeated 2800 times using every method. The classification accuracies of the proposed method, AMIs, and MSA in this situation are $96.00 \%, 100 \%$, and $95.31 \%$, respectively. The results indicate that AMIs perform best in this test, and the proposed method is similar with MSA.

The effect of adding different kinds of noises is also studied. The noise is added to the affine-transformed images before recognition.

We firstly add the salt and pepper noise with intensities varying from 0.005 to 0.03 to the transformed images.
Table 2 shows the classification accuracies of all methods in the corresponding noise degree. We can observe that the classification accuracy of AMIs decreases rapidly from noise-free condition to small noise degree. The classification accuracy decreases from $100 \%$ to less than $50 \%$ when the noise intensity is 0.010 . MSA performs much better than AMIs, but the results are not satisfying. To large noise degrees, the proposed method keeps high accuracies all the time.

In addition, we add the Gaussian noise with zero mean and different variance varying from 0.005 to 0.03 to the transformed images. Table 3 shows the classification accuracies of all methods in the corresponding noise degree. The results indicate that AMIs and MSA are much more sensitive to Gaussian noise than salt and pepper noise. However, the classification accuracies of the proposed method outperform AMIs and MSA in every noise degree.

The experimental results tell us that the proposed method presents better performances in noise situations. The reason may lie in that CPT is robust to noise. It was shown in [29] that Radon transform is quite robust to noise. We can similarly show that GC derived by CPT from the object is robust to additive noise as a result of summing pixel values to generate GC. 

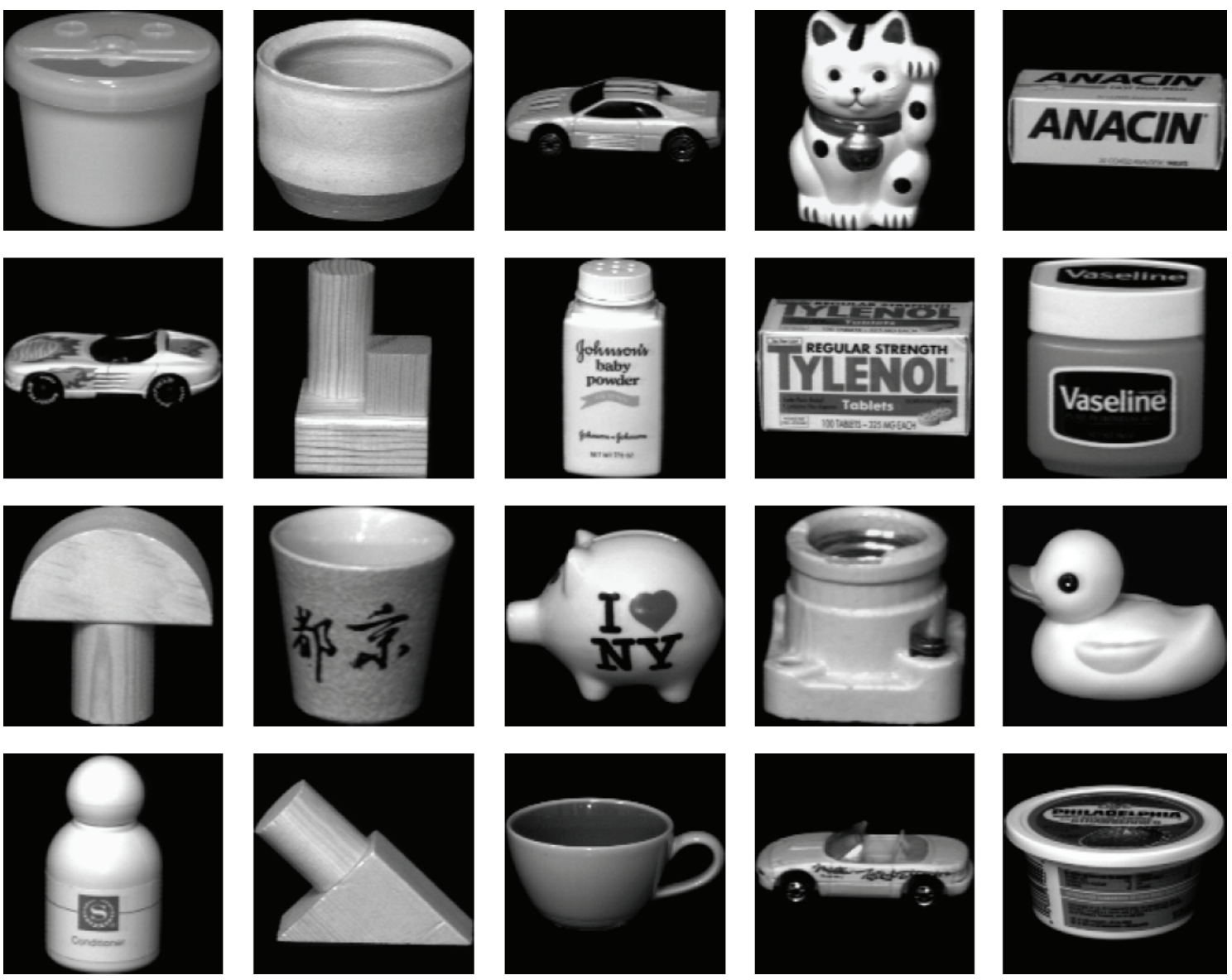

FIgUre 6: Columbia Coil-20 image database.

TABLE 2: Classification accuracies of AMIs, MSA, and the proposed method for images in Figure 6 in case of affine transformation and different intensities of salt and pepper noise.

\begin{tabular}{lccccccc}
\hline Noise degree & 0 & 0.005 & 0.010 & 0.015 & 0.020 & 0.025 & 0.030 \\
\hline AMIs & $100 \%$ & $55.04 \%$ & $43.29 \%$ & $35.93 \%$ & $31.96 \%$ & $26.75 \%$ & $23.04 \%$ \\
MSA & $95.31 \%$ & $88.10 \%$ & $74.18 \%$ & $62.14 \%$ & $53.74 \%$ & $47.02 \%$ & $42.08 \%$ \\
Our method & $96.00 \%$ & $95.07 \%$ & $93.18 \%$ & $89.25 \%$ & $83.32 \%$ & $77.61 \%$ & $72.54 \%$ \\
\hline
\end{tabular}

TABLE 3: Classification accuracies of AMIs, MSA, and the proposed method for images in Figure 6 in case of affine transformation and different intensities of Gaussian noise.

\begin{tabular}{lccccccc}
\hline Noise degree & 0 & 0.005 & 0.010 & 0.015 & 0.020 & 0.025 & 0.030 \\
\hline AMIs & $100 \%$ & $32.50 \%$ & $26.04 \%$ & $21.82 \%$ & $19.54 \%$ & $17.64 \%$ & $16.05 \%$ \\
MSA & $95.31 \%$ & $57.04 \%$ & $45.82 \%$ & $40.79 \%$ & $37.39 \%$ & $34.78 \%$ & $32.79 \%$ \\
Our method & $96.00 \%$ & $85.57 \%$ & $74.89 \%$ & $63.07 \%$ & $51.71 \%$ & $42.89 \%$ & $34.93 \%$ \\
\hline
\end{tabular}

\section{Conclusions}

In this paper, affine invariant features are extracted by using fractal. A closed curve, which is called GC, is derived from the original input pattern by employing CPT. Due to shearing, affine invariant features cannot be extracted from GC directly. To address this problem, a group of curves (which are called shift curves) are constructed from the obtained GC. Fractal dimensions of these curves can readily be computed and constitute a new feature vector for the original pattern. The derived feature vector is used for object classification tasks. Several experiments have been conducted to evaluate the performance of the proposed method.

Although satisfying results have been achieved in object classification tasks, some remarks should be made. The performance of CPT depends strongly on the accuracy calculation of the centroid. We are working towards developing 
method without the centroid. Furthermore, some characteristics of CPT should be further studied.

\section{Acknowledgments}

This work was supported in part by the National Science Foundation under Grant 60973157, and Ming Li thanks the supports in part by the 973 plan under the project Grant no. 2011CB302800 and by the National Natural Science Foundation of China under the project Grant nos. 61272402, 61070214 , and 60873264 .

\section{References}

[1] Q. M. Tieng and W. W. Boles, "Wavelet-based affine invariant representation: a tool for recognizing planar objects in $3 \mathrm{D}$ space," IEEE Transactions on Pattern Analysis and Machine Intelligence, vol. 19, no. 8, pp. 846-857, 1997.

[2] M. R. Daliri and V. Torre, "Robust symbolic representation for shape recognition and retrieval," Pattern Recognition, vol. 41, no. 5, pp. 1799-1815, 2008.

[3] P. L. E. Ekombo, N. Ennahnahi, M. Oumsis, and M. Meknassi, "Application of affine invariant fourier descriptor to shape based image retrieval," International Journal of Computer Science and Network Security, vol. 9, no. 7, pp. 240-247, 2009.

[4] X. Gao, C. Deng, X. Li, and D. Tao, "Geometric distortion insensitive image watermarking in affine covariant regions," IEEE Transactions on Systems, Man and Cybernetics C, vol. 40, no. 3, pp. 278-286, 2010.

[5] M. I. Khalil and M. M. Bayoumi, "A dyadic wavelet affine invariant function for 2D shape recognition," IEEE Transactions on Pattern Analysis and Machine Intelligence, vol. 23, no. 10, pp. 1152-1164, 2001.

[6] M. I. Khalil and M. M. Bayoumi, "Affine invariants for object recognition using the wavelet transform," Pattern Recognition Letters, vol. 23, no. 1-3, pp. 57-72, 2002.

[7] G. Liu, Z. Lin, and Y. Yu, "Radon representation-based feature descriptor for texture classification," IEEE Transactions on Image Processing, vol. 18, no. 5, pp. 921-928, 2009.

[8] R. Matungka, Y. F. Zheng, and R. L. Ewing, "Image registration using adaptive polar transform," IEEE Transactions on Image Processing, vol. 18, no. 10, pp. 2340-2354, 2009.

[9] Y. Wang and E. K. Teoh, "2D affine-invariant contour matching using B-spline model," IEEE Transactions on Pattern Analysis and Machine Intelligence, vol. 29, no. 10, pp. 1853-1858, 2007.

[10] F. Mai, C. Q. Chang, and Y. S. Hung, "A subspace approach for matching 2D shapes under affine distortions," Pattern Recognition, vol. 44, no. 2, pp. 210-221, 2011.

[11] M. Gong, H. Li, and W. Cao, "Moment invariants to affine transformation of colours," Pattern Recognition Letters, vol. 34, no. 11, pp. 1240-1251, 2013.

[12] X. Song, D. Muselet, and A. Tremeau, "Affine transforms between image space and color space for invariant local descriptors," Pattern Recognition, vol. 46, no. 8, pp. 2376-2389, 2013.

[13] D. Zhang and G. Lu, "Review of shape representation and description techniques," Pattern Recognition, vol. 37, no. 1, pp. 1-19, 2004.

[14] E. Rahtu, A multiscale framework for affine invariant pattern recognitionand registration [Ph.D. thesis], University of OULU, Oulu, Finland, 2007.
[15] R. Veltkamp and M. Hagedoorn, "State-of the art in shape matching," Tech. Rep. UU-CS-1999, 1999.

[16] I. Weiss, "Geometric invariants and object recognition," International Journal of Computer Vision, vol. 10, no. 3, pp. 207-231, 1993.

[17] Y. Y. Tang, Y. Tao, and E. C. M. Lam, "New method for extractionbased on fractal behavior," Pattern Recognition, vol. 35, pp. 1071-1081, 2002.

[18] B. B. Mandelbrot, The Fractal Geometry of Nature, W. H. Freeman, San Francisco, Calif, USA, 1982.

[19] B. B. Chaudhuri and N. Sarkar, "Texture segmentation using fractal dimension," IEEE Transactions on Pattern Analysis and Machine Intelligence, vol. 17, no. 1, pp. 72-77, 1995.

[20] Y. Y. Tang, J. Liu, H. Ma, and B. Li, "Two-dimensional wavelet transform indocument analysis," in Proceedings of the 1st International Conferenceon Multimodal Interface, pp. 274-279, Beijing, China, October 1996.

[21] D. Cyganski and R. F. Vaz, "A linear signal decomposition approach to affineinvariant contour identification," in Intelligent Robots and Computer Vision X, vol. 1607 of Proceedings of SPIE, pp. 98-109, 1991.

[22] K. Arbter, W. E. Snyder, H. Burkhardt, and G. Hirzinger, "Application of affine-invariant Fourier descriptors to recognition of 3-D objects," IEEE Transactions on Pattern Analysis and Machine Intelligence, vol. 12, no. 7, pp. 640-647, 1990.

[23] M. Yang, K. Kpalma, and J. Ronsin, "Affine invariance contour desciptor based on the equal area normalization," IAENG International Journal of Applied Mathematics, vol. 36, no. 2, paper 5, p. 6, 2007.

[24] K. Falconer, Fractal Geometry. Mathematical Foundations and Applications, John Wiley \& Sons, Chichester, UK, 1990.

[25] G. A. Edgar, Measure, Topology, and Fractal Geometry, Undergraduate Texts in Mathematics, Springer, New York, NY, USA, 1990.

[26] J. Flusser and T. Suk, "Pattern recognition by affine moment invariants," Pattern Recognition, vol. 26, no. 1, pp. 167-174, 1993.

[27] E. Rahtu, M. Salo, and J. Heikkilä, "Affine invariant pattern recognition using multiscale autoconvolution," IEEE Transactions on Pattern Analysis and Machine Intelligence, vol. 27, no. 6, pp. 908-918, 2005.

[28] http://wwwl.cs.columbia.edu/CAVE/software/softlib/coil-20 .php.

[29] K. Jafari-Khouzani and H. Soltanian-Zadeh, "Rotationinvariant multiresolution texture analysis using Radon and wavelet transforms," IEEE Transactions on Image Processing, vol. 14, no. 6, pp. 783-795, 2005. 


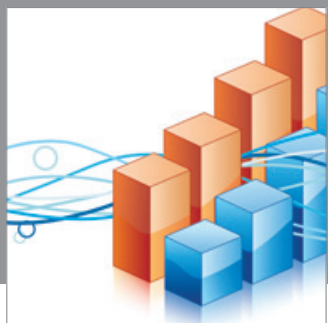

Advances in

Operations Research

mansans

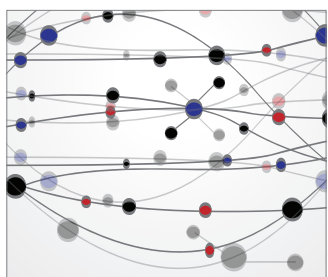

The Scientific World Journal
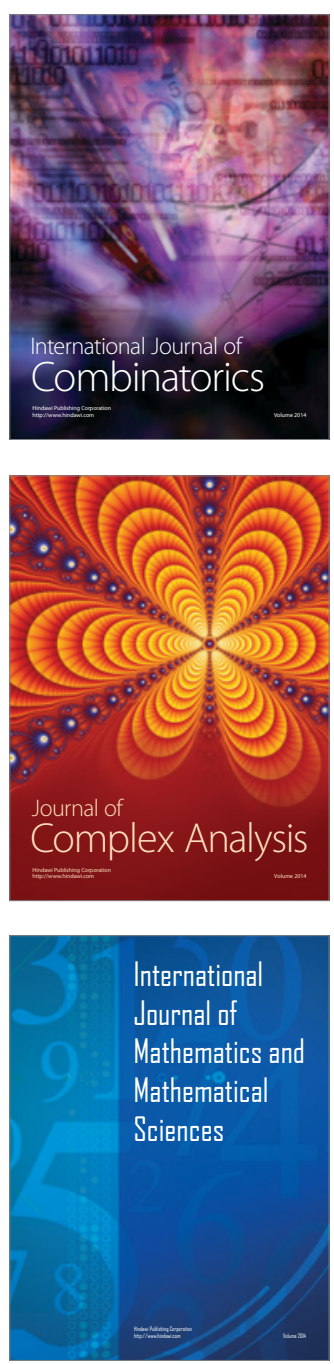
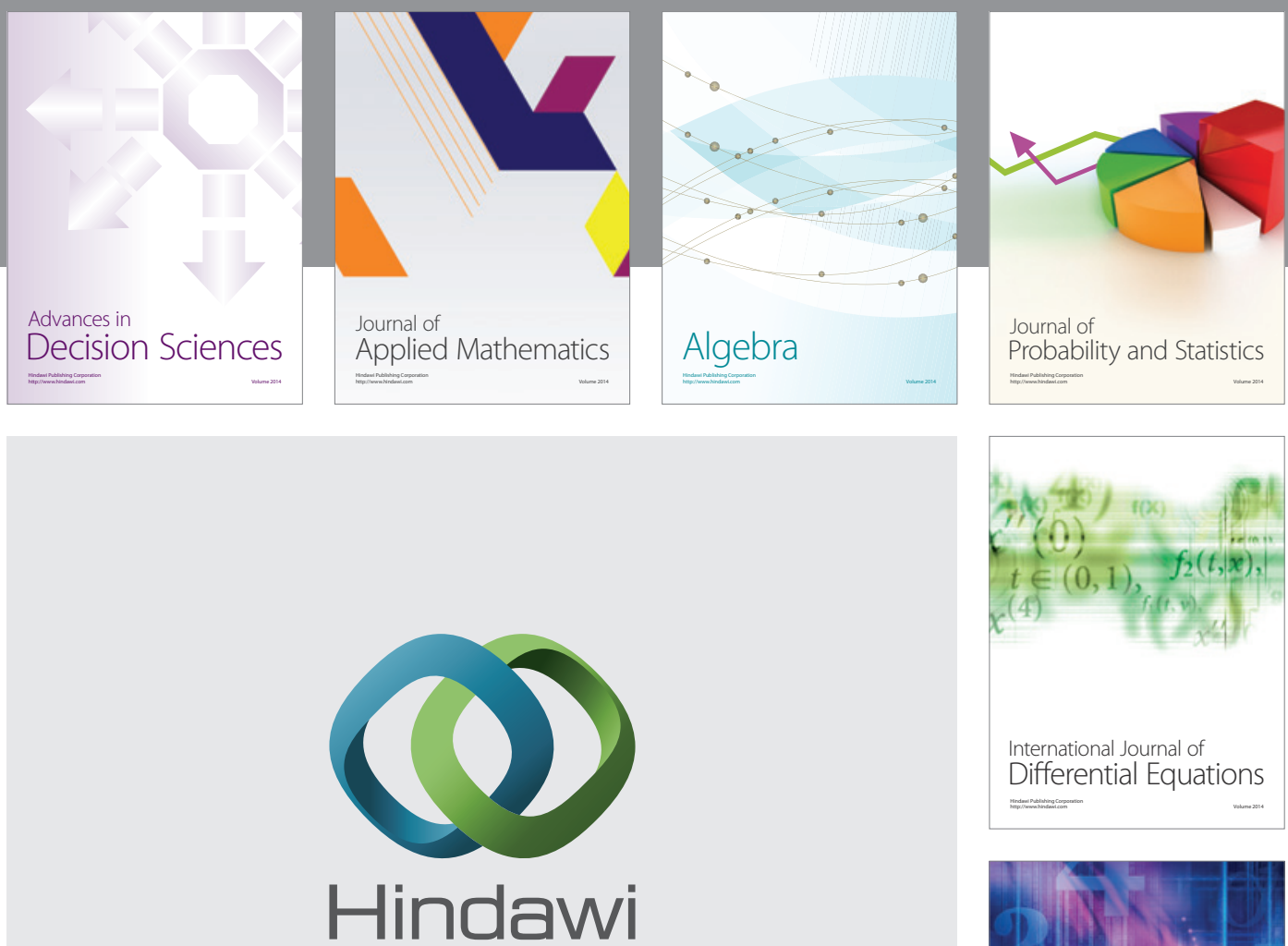

Submit your manuscripts at http://www.hindawi.com
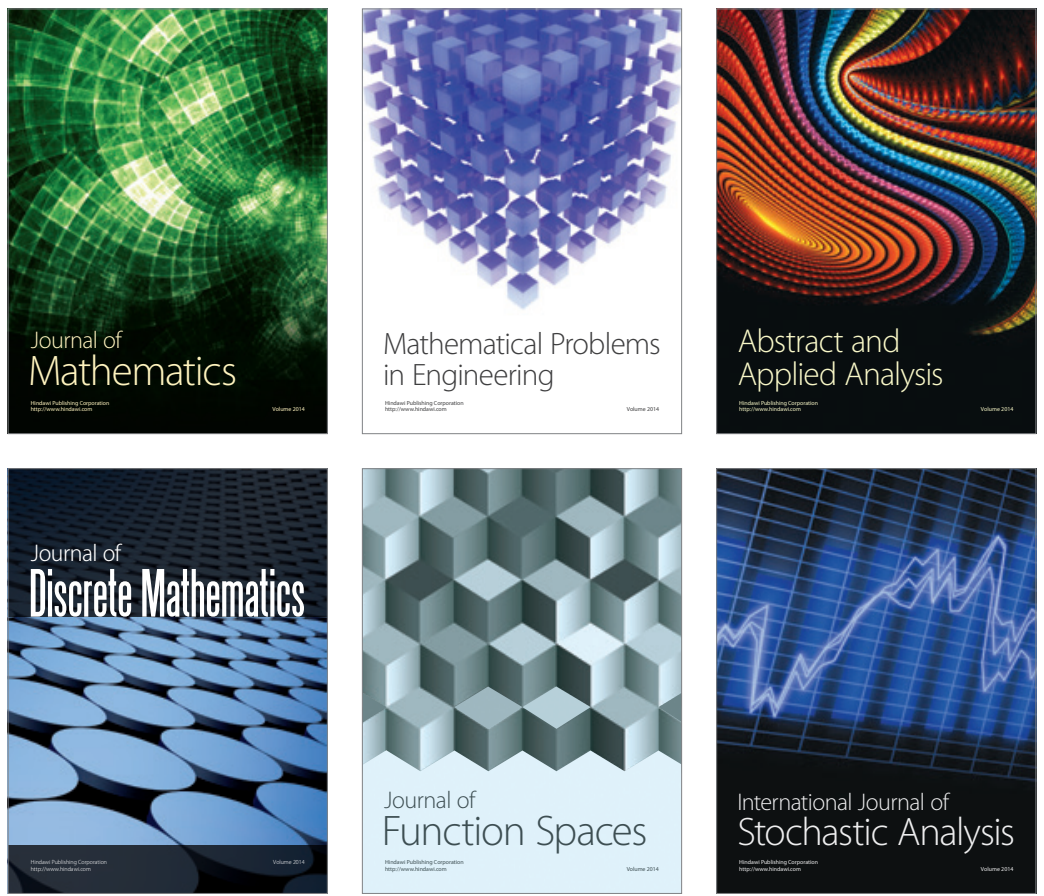

Journal of

Function Spaces

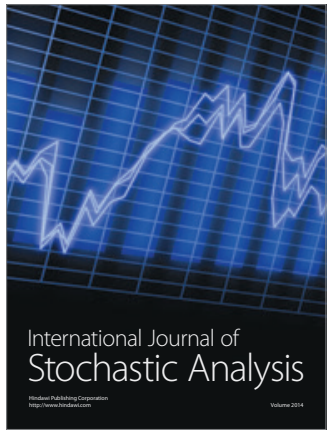

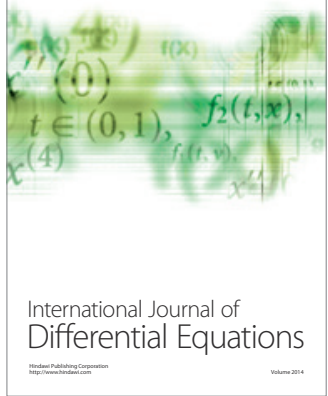
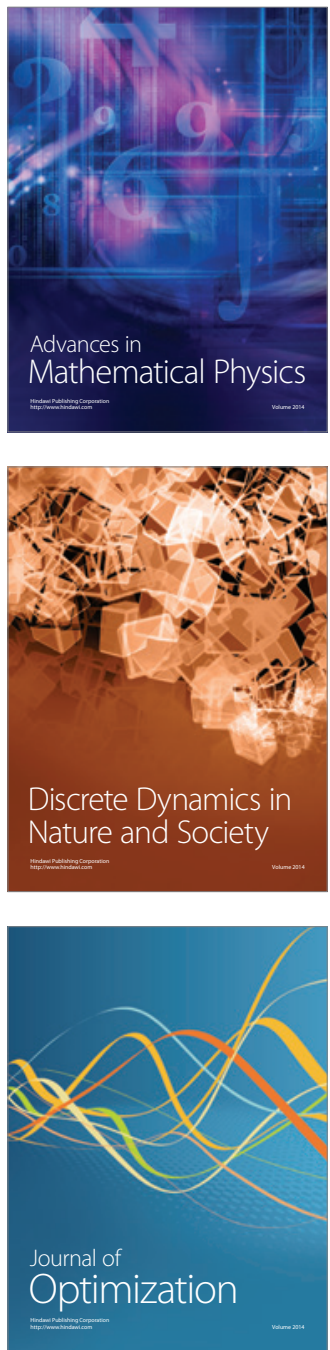